\title{
Gene Note
}

\section{Cloning of cDNAs for DELLA proteins suppressing cysteine proteinase genes in germinated cotyledons of common bean seeds}

\author{
Daisuke Yamauchi*, Masahiro Kobayashi, Tomonori Nakai, Kou Kubota \\ Department of Life Sciences, University of Hyogo, Hyogo 671-2201 Japan \\ *E-mail: dyamauch@sci.u-hyogo.ac.jp Tel: +81-79-267-4939 Fax: +81-79-267-4920
}

Received May 28, 2007; accepted October 3, 2007 (Edited by T. Mizoguchi)

\begin{abstract}
Gibberellins (GAs) are involved in the expression of cysteine proteinase genes in germinated cotyledons of common bean seeds. Because DELLA proteins are known to be transcriptional repressors mediating GA signaling, we isolated two cDNA clones encoding DELLA proteins (PvGAI1 and PvGAI2) from common bean seedlings to examine the mechanism of GA signaling involved in the expression of the proteinase genes. RT-PCR and RNA blot analyses indicated that the level of mRNA in germinated cotyledons was higher for PvGAI2 than for PvGAII. We also found that transient expression of $P v G A I 2$, but not that of $P v G A I 1$, repressed the promoter activities of GA-inducible cysteine proteinase genes, $E P-C 1$ and $C P 2$, in germinated cotyledons. These findings suggest that PvGAI2 is mainly responsible for regulating the expression of proteinase genes in germinated cotyledons. Application of a GA-biosynthesis inhibitor, prohexadione calcium to common bean seeds had little effect on the RNA level of $P v G A I 2$, although the inhibitor repressed genes for EP-C1 and $C P 2$. Because it is known that GA induces degradation of DELLA proteins, our findings suggest that the level of GA, but not the mRNA expression of PvGAI2, regulates the protein level of PvGAI2 suppressing the proteinases genes in germinated cotyledons.
\end{abstract}

Key words: $\quad$ DELLA protein, gibberellin, Phaseolus vulgaris, proteinase.

It is well known that gibberellins (GAs) induce the expression of hydrolases, including proteinases and $\alpha$ amylases, in aleurone cells of cereal seeds. GA signaling molecules have been identified using Arabidopsis mutants (Peng et al. 1997, Silverstone et al. 1998) with these studies demonstrating that the GA response mediates genes for REPRESSOR OF GA1-3 (RGA) and GA INSENSITIVE (GAI) encoding a type of transcription factor called DELLA protein. The DELLA proteins contain the conserved amino acid sequence Asp-GluLeu-Leu-Ala (D-E-L-L-A) at their N-terminal regions and act as transcriptional repressors for GA-inducible genes. The rice mutant slender ricel (slrl) shows a slender phenotype and constitutive expression of $\alpha$ amylase in its aleurone cells, and the gene responsible, SLR1, encodes a DELLA protein (Ikeda et al. 2001). Additionally, transient expression of the gene for a barley DELLA protein, SLENDER1 (SLN1), repressed an $\alpha$ amylase gene promoter in aleurone cells (Zentella et al. 2002). These findings indicate that DELLA proteins regulate the degradation of storage reserves in cereal seeds.

We previously suggested that storage protein degradation in cotyledons of common bean seeds is involved in GA biosynthesis (Yamauchi 2007), given that two GA-biosynthesis inhibitors, prohexadione calcium (PHD) and chloroethyltrimethylammonium chloride, repressed the genes encoding cysteine proteinases EPC1, CP1, CP2, CP3, LLP1, and LLP2. The expression levels of these six genes were recovered by exogenous application of $\mathrm{GA}_{3}$. However, it is still unclear whether DELLA proteins mediate GA biosynthesis and expression of the proteinase genes in germinated cotyledons. In the present study we cloned cDNAs for DELLA proteins from common bean seeds and examined whether transient expression of the proteins repressed promoter activity of the GA-inducible proteinase genes, $E P-C l$ and $C P 2$, in germinated cotyledons.

Seeds of common bean (Phaseolus vulgaris L. cv Gold Star) were obtained from Sakata Seed (Yokohama, Japan). The seeds were imbibed for $6 \mathrm{~h}$, and germinated in vermiculite at $27^{\circ} \mathrm{C}$ in darkness. PHD and $\mathrm{GA}_{3}$ were applied as described by Yamauchi (2007).

DNA was prepared from the seedlings as described by Yamauchi et al. (1989) and a total RNA fraction was prepared by the SDS-phenol method as described by Zhong et al. (1997). Poly (A) ${ }^{+}$RNA was purified from

Abbreviations: CaMV, cauliflower mosaic virus; DAI, day after imbibition; Fluc, firefly luciferase; GA, gibberellin; Luc, luciferase; LZ, leucine heptad repeat; PHD, prohexadione calcium; RACE, rapid amplification of cDNA ends; Rluc, Renilla luciferase.

This article can be found at http://www.jspcmb.jp 
the total RNA using Oligo-tex (Takara Bio, Otsu, Japan). Total RNA was denatured with glyoxal, separated on a $1.4 \%$ agarose gel, and transferred to a nylon membrane (Hybond-N, Amersham Biosciences, Buckinghamshire, UK) as described by Sambrook et al. (1989). Probe DNAs were labeled with PCR DIG labeling mix (Roche Diagnostics, Mannheim, Germany) and reacted to the membrane in a hybridization buffer as described by Church and Gilbert (1984). After hybridization, RNA was detected with DIG nucleic acid detection kit (Roche Diagnostics), except for the substrate of alkaline phosphatase, and its activity was detected with CDP star detection reagent (Amersham Biosciences).

Fragments containing genes encoding the DELLA proteins were obtained from genomic DNA using PCR with the degenerate primers described by Bassel et al. (2004). To obtain entire sequences of the cDNAs, $5^{\prime}$ - and 3'-rapid amplification of cDNA ends (RACE) were carried out using the GeneRacer Kit (Invitrogen, Carlsbad, CA, USA). Sequences of PvGAI1 and PvGAI2 cDNAs were amplified by RT-PCR from the poly (A) ${ }^{+}$ RNA of seedlings at 3 days after imbibition (DAIs). The amplified fragments were cloned into pCR2.1 (Invitrogen), and the resultant plasmids were named pGAI1 and pGAI2. Entire coding regions of PvGAI1 and PvGAI2 were amplified from pGAI1 and pGAI2 using the Pyrobest DNA polymerase (Takara Bio) and cloned into pENTR-TOPO (Invitrogen); the resultant plasmids were narmed pENTR-GAI1 and pENTR-GAI2, respectively. Sequences of PvGAI1 and PvGAI2 cDNAs were moved to the destination vector pUGWB2 (which was kindly donated by Dr. Nakagawa, Shimane University) using LR ClonaseII (Invitrogen), and the obtained plasmids were named p35S-GAI1 and p35SGAI2, respectively. To delete the $55 \mathrm{~N}$-terminal amino acids and 7 C-terminal amino acids of PvGAI2, Met 56 and Trp 589 (TGG) were changed to the initiation codon and the stop codon (TGA) by PCR, respectively, and the resultant fragment was cloned into pUGWB2 as described above; the obtained plasmids were named p35S-GAI2nd and p35S-GAI2cd, respectively. To delete the 17 amino acids including the DELLA domain, an AatII site was created at Asp 41 by PCR using pENTRGAI2 as the template and $5^{\prime}$-CACCTTCCGACATGGCAGACGTCGCTCAGA-3' and 5'- GGAAGCTTTCACGCAGAGGTGGCGATGAGA-3' as the primers. The cDNA fragments were then cut with AatII and ligated to the plasmid AatII site at Asp 58. The mutated PvGAI2 cDNA fragment was cloned into pUGWB2 as described above, and the resultant plasmid was named p35SGAI2dd.

A reporter plasmid was constructed by cutting pDO432 (Ow et al. 1986) with BamHI and SacI, and the obtained fragment containing the firefly luciferase (Fluc) gene was ligated to pBI221 (Jefferson et al. 1987) that had also been cut by BamHI and SacI; the resultant plasmid was named p35S-Fluc. The promoter region from the $\mathrm{EP}-\mathrm{Cl}$ gene was amplified as described by Terasaki et al. (1995) and fused to the Fluc coding region of p35S-Fluc, with the resultant plasmid named pEPC1Fluc. The promoter region from positions -2302 to +11 of the $C P 2$ gene was amplified to create a $B a m H I$ site at +12 of the gene by PCR, and the amplified fragment was inserted into HindIII and BamHI sites of p35S-Fluc; the resultant plasmid was named $\mathrm{pCP} 2$-Fluc.

To normalize Fluc activity, p221-Rluc (Kitakura et al. 2002) - in which the cauliflower mosaic virus (CaMV) 35S RNA promoter is fused to the Renilla luciferase (Rluc) gene-was used as an internal control. All plasmids were mixed in equal amounts $(1 \mu \mathrm{g}$ each per $1 \mathrm{mg}$ of gold particles). Reporters and effectors were introduced into germinated cotyledons of common bean seeds at 3 DAIs by particle bombardment as described by Yamauchi (1997). After bombardment, the cotyledons were incubated on wet filter paper at $27^{\circ} \mathrm{C}$ in darkness for $24 \mathrm{~h}$. Luciferase (Luc) activities were measured using the dual luciferase reporter assay kit (Promega, Madison, WI, USA).

We cloned two PCR fragments using genome DNA as a template. These sequences are similar to those of GmGAI1 and GmGAI2 (Bassel et al. 2004), and the corresponding genes were named $P v G A I 1$ and $P v G A I 2$, respectively. We cloned the entire cDNA sequences with the RACE method using primers designed from the sequences of the fragments. Sequences of PvGAII and PvGAI2 cDNAs were 2090 and 2051 nucleotide residues without poly(A) tails that encoded 517 and 596 amino acid residues, respectively (Accession Nos. AB304457 and $\mathrm{AB} 304458)$. These amino acid sequences are homologous to previously reported DELLA proteins, and are compared to that of Arabidopsis GAI in Figure 1A. The amino acid sequences of both PvGAI1 and PvGAI2 included DELLA, TVHYNP, S/T/V, leucine heptad repeat (LZ), VHIID, and SAW domains that are all conserved in DELLA proteins (Figure 1B; Itoh et al. 2002, Pysh et al. 1999). However, the N-terminal half regions exhibited low identities between PvGAI1 and PvGAI2, which is due to the $\mathrm{S} / \mathrm{T} / \mathrm{V}$ domain being longer in PvGAI2 than in PvGAI1 (Figure 1A).

We examined the levels of PvGAII and PvGAI2 mRNAs in germinated cotyledons of common bean seeds at 3 DAIs by RT-PCR (Figure 2A). Although $E P$ C1 mRNA was detected after 25 cycles, PvGAI2 mRNA was not detected until 30 cycles, at which point $P v G A I I$ mRNA was still not detected. These results suggest that the gene encoding $P v G A I 1$ is expressed at lower levels than the gene encoding $P v G A I 2$ in germinated cotyledons. We also performed an RNA blot analysis of the two genes during seed germination (Figure 2B). PvGAI2 mRNA appeared at 1 DAI and its level was 




B

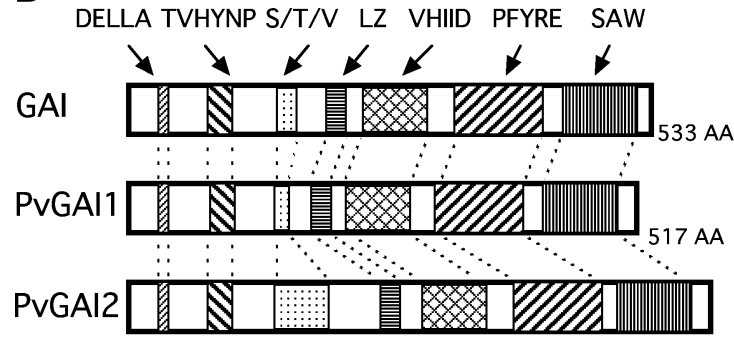

596 AA

Figure 1. Comparison of the primary structure of common bean DELLA proteins with Arabidopsis GAI. (A) Alignment of amino acid sequences of GAI, PvGAI1 and PvGAI2. Identical amino acids are indicated as white letters on the black backgrounds. The conserved domains of the DELLA proteins DELLA, TVHYNP, S/T/V, LZ, VHIID, PFYRE, and SAW are indicated above the amino acid sequences. (B) Schematic representation of the structures of GAI. PvGAI1 and PvGAI2. The conserved domains of the DELLA proteins DELLA, TVHYNP, S/T/V, LZ, VHIID, PFYRE, and SAW are indicated as shaded boxes. The numbers of amino acid residues of GAI, PvGAI1 and PvGAI2 are indicated on the right. AA. amino acids.

maintained until 3 DAIs. $E P$ - $C 1$ and $C P 2$ mRNAs were detected at $1 \mathrm{DAI}$ and their levels were increased at 3 DAIs. In contrast, only a very weak band corresponding to $P v G A I 1$ mRNA was found from 1 DAI to 3 DAIs. The GA-biosynthesis inhibitor PHD is known to repress $E P$ $C 1$ and CP2 (Yamauchi 2007). We examined the effect
A
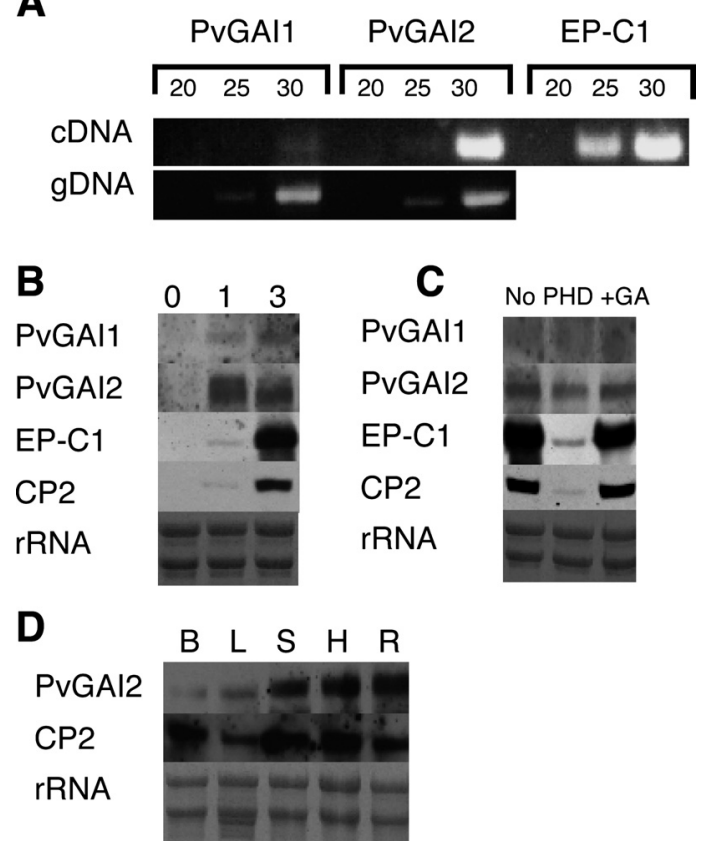

Figure 2. Analysis of expression levels of $P v G A I 1$ and $P v G A I 2$ in common bean plants. (A) RT-PCR detection of $P v G A I 1$ and $P v G A I 2$ mRNAs in total RNA from germinated cotyledons. cDNA was synthesized from total RNA extracted from germinated cotyledons at 3DAIs (cDNA). PCR was applied to a genomic DNA of common bean as a positive control (gDNA). Each number at the top of the panel indicates the number of PCR cycles. The following primers were used for mRNA detection: PvGAII 5'-GTGGTTGTTGACTCCCAGGAGAA-3' and 5'-CGTAGAagtgaAtCTGGAGAGAGT-3'; PvGAI2 (5'TTGACTCACAGGAAGCCGGCGT-3' and 5'- GTCGGAGAGAGAGGATTCAAGAGT-3'; $\quad$ EP-Cl 5'-GGAGGCATAACAACAGAAAGCAATT-3' and 5' ${ }^{\prime}$-CTACAATCACCAGTAAATACTCCCT-3'. Thermal cycling comprised denaturing at $94^{\circ} \mathrm{C}$ for 1 min, annealing at $55^{\circ} \mathrm{C}$ at $1 \mathrm{~min}$ and extension at $72^{\circ} \mathrm{C}$ for $1 \mathrm{~min}$. The amplified fragments were separated on a $2 \%$ agarose gel and stained with ethidium bromide. (B) RNA blot analysis of PvGAI1 and PvGAI2 mRNAs in germinated cotyledons. Total RNA fractions were extracted from germinated cotyledons of seeds. Numbers at the top of panel indicate DAIs. (C) RNA blot analysis of effect of PHD on expression levels of PvGAII and PvGAI2. Common bean seeds were allowed to imbibe water (No), $10 \mu \mathrm{M}$ PHD (PHD), or $10 \mu \mathrm{M}$ PHD and $10 \mu \mathrm{M}$ $\mathrm{GA}_{3}$ (+GA). (D) RNA blot analysis of expression of PvGAI2 in various tissues of seedlings. Common bean plants were grown in a phytotron with continuous light for 7 DAIs. Buds (B), leaves (L), stems $(\mathrm{S})$, hypocotyls $(\mathrm{H})$, and roots $(\mathrm{R})$ were collected from the plants. $P v G A I 1$ and EP-Cl mRNAs were not detected in total RNA from the tissues of the seedlings. Probes of cDNA for PvGAII (PvGAI1), $P v G A I 2$ (PvGAI2), EP-Cl (EP-C1) and CP2 (CP2) were used for hybridization. rRNA was stained with methylene blue (rRNA).

of PHD on the expression levels of $P v G A I 1$ and $P v G A I 2$. Exogenously applied PHD decreased the levels of $E P-C 1$ and $C P 2$ mRNAs, and this was prevented by the addition of $\mathrm{GA}_{3}$ (Figure 2C). In contrast, PHD had little effects on the levels of $P v G A I 1$ and $P v G A I 2$ mRNAs. These results indicated that the levels of $P v G A I 1$ and $P v G A I 2$ mRNAs are not correlated with those of $E P-C 1$ and $C P 2$. We predict that GA induces breakdown of DELLA proteins and activates proteinase genes in germinated cotyledons of common bean seeds. Immunological detection of 
PvGAI2 in germinated cotyledons is required to test this prediction. In addition, our result suggests that the mRNA level of $P v G A I 2$ increased rapidly until 1 DAI (Figure 2B). This result may be involved in spatial regulation of proteinase genes in cotyledons. Because it is known that mobilization of storage protein starts at the farthest region from the vascular bundle in the cotyledon of legume plants (Toyooka et al. 2000), proteinase genes may be activated in a similar manner to the mobilization. We predict that synthesis of PvGAI2 at early germination is required to repress proteinase genes around the

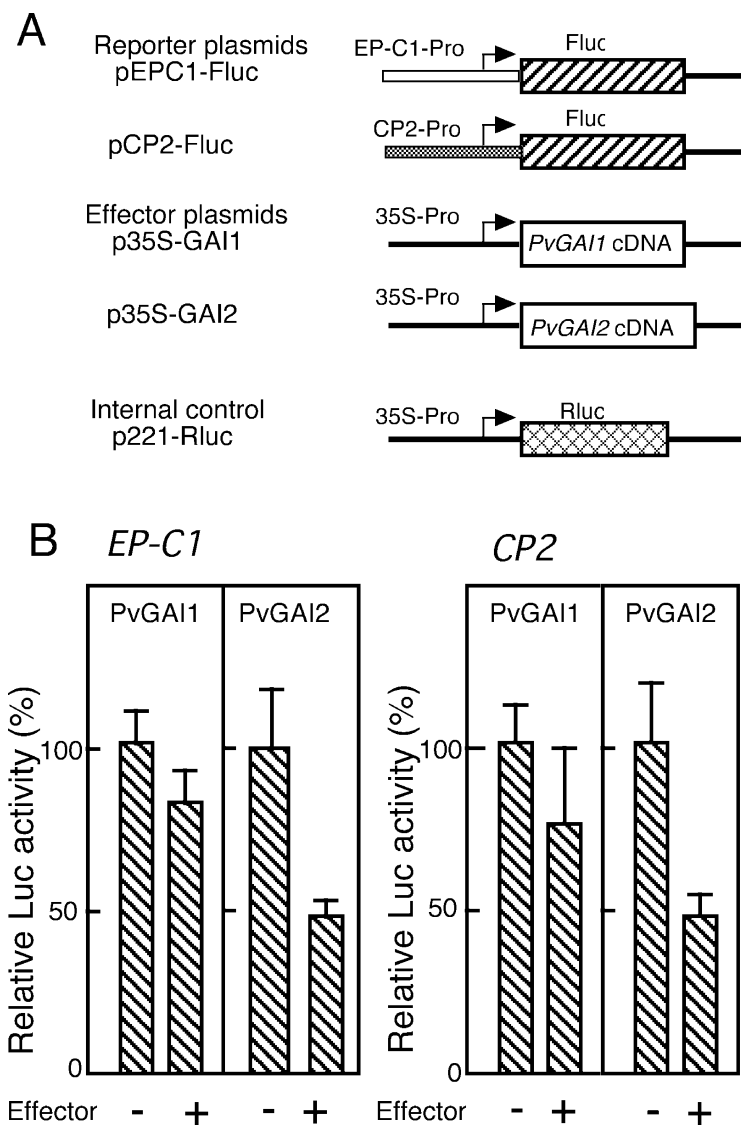

Figure 3. Repression of the promoter activities of $E P-C 1$ and $C P 2$ by the transient expression of PvGAI2. (A) Structures of reporter plasmids, effector plasmids and an internal control plasmid. The reporter plasmids, pEPC1-Fluc and pCP2-Fluc contain the Fluc gene (shaded box) fused to the $E P-C 1$ promoter region from -1173 to +15 (EP-C1-Pro) and to the $C P 2$ promoter region from -2302 to +11 (CP2-Pro). The effector plasmids include PvGAII or PvGAI2 cDNA driven by CaMV 35S RNA promoter (35S-Pro). The internal control plasmid, p221-Rluc, contains the Rluc gene (hatched box) fused to CaMV 35S RNA promoter. (B) Effects of transient expression of $P v G A I 1$ and $P v G A I 2$ on Fluc activity driven from the promoters of $E P$ $C 1$ and $C P 2$. We delivered the reporter plasmid, pEPC1-Fluc (left panel; $E P-C 1$ ) or pCP2-Fluc (right panel; $C P 2$ ), in the presence $(+)$ or absence (-) of the effector, p35S-GAI1 (PvGAI1) or p35S-GAI2 (PvGAI2), into the cotyledons of common bean seeds at 3 DAIs using particle bombardments. Relative Luc activity was calculated by dividing the Fluc to Rluc ratio from each construct by the Fluc to Rluc ratio from $\mathrm{pEPC1}$-Fluc or pCP2-Fluc. Data are means and standard errors from four independent bombardments. The promoter activities did not differ significantly between the between presence and absence of the effector, p35S-GAI1. vascular bundle. Immunohistochemical detection of PvGAI2 is needed to test this hypothesis.

RNAs from tissues of seedlings were analyzed to examine tissue-specific expression of the two DELLA proteins (Figure 2D). The bands corresponding to both $P v G A I 1$ and $E P-C 1$ mRNAs were not visible (data not shown). In contrast, bands corresponding to $P v G A I 2$ and $C P 2$ mRNAs were found in RNA extracted from all tissues of the seedlings (Figure 2D). It is therefore possible that PvGAI2, but not PvGAI1, regulates the GA response in vegetative tissues.

DELLA proteins are known to act as transcriptional repressor. We examined whether PvGAI1 and PvGAI2 repressed the promoter activities of the GA-inducible cysteine proteinase genes $E P-C 1$ and $C P 2$ using a transient expression system with particle bombardment. The transient expression of $P v G A I 2$ decreased the Fluc activity derived from the promoters of $E P-C 1$ and $C P 2$ (Figure 3). However, PvGAIl did not repress the activities of the two promoters, as indicated by there being no statistical differences between the presence and absence of the effector, p35S-GAI1. The mRNA level in germinated cotyledons was higher for PvGAI2 than for PvGAII (Figure 2A, B). These results suggest that PvGAI2, but not PvGAI1, regulates GA-inducible gene expression in germinated cotyledons of common bean seeds.

We examined whether or not the DELLA domain and the C-terminal region of PvGAI2 were required for repression of $\mathrm{EP}-\mathrm{Cl}$ promoter activity (Figure 4). Deletion of the seven C-terminal amino acids (p35SGAI2cd) resulted in PvGAI2 losing its ability to repress $\mathrm{EP}-\mathrm{Cl}$ promoter activity. This finding mirrors the results of a previous analysis of the rice DELLA protein SLR1 (Ikeda et al. 2001). Both deletion of the N-terminal region containing a DELLA domain (p35S-GAI2nd) and an internal deletion of 19 amino acids containing a DELLA domain (p35S-GAI2dd) resulted in $25 \%$ and $43 \%$ increases, respectively, in $E P-C 1$ promoter activity.

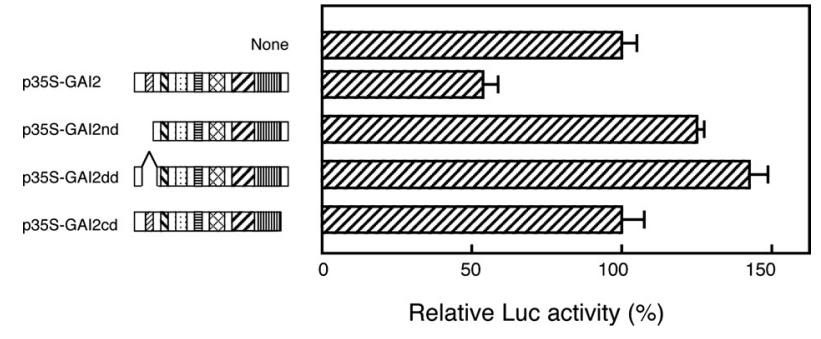

Figure 4. Effect of deletion of PvGAI2 amino acids on expression of the $E P-C l$ gene. The effectors are listed on the left. Shaded boxes in schematic presentations of PvGAI2 coding regions are described in Figure 1B. We delivered the reporter plasmid, pEPC1-Fluc, and the effectors into the cotyledons of common bean seeds at 3 DAIs using particle bombardments. Relative Luc activity was calculated as described in the legend of Figure 3. Data are means and standard errors from four independent bombardments. 
Ueguchi-Tanaka et al. (2005) reported that the Nterminal DELLA domain is involved in GA receptor recognition, and that its deletion blocks the GA response. However, this finding is not consistent with our results. If the deletion of the DELLA domain results in PvGAI2 losing its ability to repress GA-inducible genes, it is probable that the mutated PvGAI2 forms a heterodimer with endogenous PvGAI2, and that a decrease in endogenous PvGIA2 homodimer affects EP-C1 promoter activity.

\section{Acknowledgements}

The authors thank Dr. Ito (Nagoya University) for the gift of plasmid p221-luc, Dr. Nakagawa (Shimane University) for the gift of plasmid pUGWB2 and Ms. Marukawa (University of Hyogo) for constructing p35S-GAI1. This work was supported by grants from the Himeji Institute of Technology and University of Hyogo.

\section{References}

Bassel GW, Zielinska E, Mullen RT, Bewley JD (2004) Downregulation of DELLA genes is not essential for germination of tomato, soybean, and Arabidopsis seeds. Plant Physiol 136: 2782-2789

Church GM, Gilbert W (1984) Genomic sequencing. Proc Natl Acad Sci USA 81: 1991-1995

Ikeda A, Ueguchi-Tanaka M, Sonoda Y, Kitano H, Koshioka M, Futsuhara Y, Matsuoka M, Yamaguchi J (2001) slender rice, a constitutive gibberellin response mutant, is caused by a null mutation of the SLR1 gene, an ortholog of the height-regulating gene GAI/RGA/RHT/D8. Plant Cell 13: 999-1010

Itoh H, Ueguchi-Tanaka M, Sato Y, Ashikari M, Matsuoka M (2002) The gibberellin signaling pathway is regulated by the appearance and disappearance of SLENDER RICE1 in nuclei. Plant Cell 14: 57-70

Jefferson RA, Kavanagh TA, Bevan MW (1987) GUS fusions: $\beta$ glucuronidase as a sensitive and versatile gene fusion marker in higher plants. EMBO J 6: 3901-3907

Kitakura S, Fujita T, Ueno Y, Terakura S, Wabiko H, Machida Y (2002) The protein encoded by oncogene $6 b$ from Agrobacterium tumefaciens interacts with a nuclear protein of tobacco. Plant Cell 14: 451-463

Ow DW, Wood KV, DeLuca M, de Wet JR, Helinski DR, Howell SH (1986) Transient and stable expression of the firefly luciferase gene in plant cells and transgenic plants. Science 234: 856-859

Peng J, Carol P, Richards DE, King KE, Cowling RJ, Murphy GP, Harberd NP (1997) The Arabidopsis GAI gene defines a signaling pathway that negatively regulates gibberellin responses. Genes Dev 11: 3194-3205

Pysh LD, Wysocka-Diller JW, Camilleri C, Bouchez D, Benfey PN (1999) The GRAS gene family in Arabidopsis: sequence characterization and basic expression analysis of the SCARECROW-LIKE genes. Plant J 18: 111-119

Sambrook J, Fritsch EF, Maniatis T (1989) Molecular Cloning. Cold Spring Harbor Laboratory Press, New York

Silverstone AL, Ciampaglio CN, Sun T (1998) The Arabidopsis $R G A$ gene encodes a transcriptional regulator repressing the gibberellin signal transduction pathway. Plant Cell 10: 155-169

Terasaki Y, Yamauchi D, Morikawa H, Minamikawa T (1995) Influence of axis removal on the expression of a gene for a cysteine endopeptidase (EP-C1) in French bean cotyledons during germination. Plant Cell Physiol 36: 537-541

Toyooka K, Okamoto T, Minamikawa T (2000) Mass transport of proform of a KDEL-tailed cysteine proteinase (SH-EP) to protein storage vacuoles by endoplasmic reticulum-derived vesicle is involved in protein mobilization in germinating seeds. J Cell Biol 148: 453-463

Ueguchi-Tanaka M, Ashikari M, Nakajima M, Itoh H, Katoh E, Kobayashi M, Chow TY, Hsing YIC, Kitano H, Yamaguchi I, Matsuoka M (2005) GIBBERELLIN INSENSITIVE DWARF1 encodes a soluble receptor for gibberellin. Nature 437: 693-698

Yamauchi D (1997) Interaction of seed embryo factors with the $5^{\prime}$ upstream region of seed storage $7 \mathrm{~S}$ globulin gene from Canavalia gladiata. Plant Sci 126: 163-172

Yamauchi D (2007) Hormonal regulation of the expression of cysteine proteinase genes in germinated cotyledons of common bean seeds. Plant Biotechmol 24: 231-235

Yamauchi D, Nakamura K, Asahi T, Minamikawa T (1989) Nucleotide sequence of cDNA for concanavalin A from Canavalia gladiata seeds. Plant Cell Physiol 30: 147-150

Zentella R, Yamauchi D, Ho TH (2002) Molecular dissection of the gibberellin/abscisic acid signaling pathways by transiently expressed RNA interference in barley aleurone cells. Plant Cell 14: 2289-2301

Zhong P-Y, Tanaka T, Yamauchi D, Minamikawa T (1997) A 28kilodalton pod storage protein of French bean plants. Purification, characterization, and primary structure. Plant Physiol 113: 479-485 